\title{
Short communication: Endoplasmic reticulum stress gene network expression in bovine mammary tissue during the lactation cycle ${ }^{1}$
}

\author{
G. Invernizzi, ${ }^{\dagger} \dagger$ A. Naeem, ${ }^{*}$ and J. J. Loor ${ }^{2}$ \\ *Mammalian NutriPhysioGenomics, Division of Nutritional Sciences, 498 Animal Science Laboratory, Department of Animal Sciences, \\ University of Illinois, Urbana 61801 \\ †Department of Veterinary Science and Technology for Food Safety, Faculty of Veterinary Medicine, University of Milan, Via Celoria 10, \\ Milan 20133, Italy
}

\begin{abstract}
The endoplasmic reticulum (ER) has a crucial role in cellular metabolism. Recent studies in nonruminants discovered that components of the ER stress pathway, induced during the unfolded protein response, play critical roles in regulating lipogenesis. The bovine mammary gland faces extreme metabolic stress at the onset of lactation due primarily to the increase in flux through pathways associated with milk fat and protein synthesis. Our objective was to study, via quantitative real-time PCR, the expression of the ER stress pathway components P58IPK, PERK, XBP1, ATF4, ATF3, ATF6, CHOP, MBTPS1, GRP94, and BiP in mammary tissue ( $\mathrm{n}=7$ cows $\times 5$ time points) collected at -15 , $1,15,60$, and $240 \mathrm{~d}$ relative to parturition. Expression of P58IPK and ATF 4 increased to a peak at d 60, followed by a decrease by d 240 postpartum. Despite the decrease in expression by $240 \mathrm{~d}$, P58IPK remained higher than prepartal levels $(\mathrm{d}-15)$. Expression patterns of ATF3 and $C H O P$ were similar and peaked at d 15, followed by a decrease through d 240, at which point $C H O P$ expression was still greater than prepartal levels. The sharp increase in milk production postpartum (d 15) as well as apoptosis during late lactation $(240 \mathrm{~d})$ may have induced a pseudo unfolded protein response state. This is supported by the similar expression patterns of P58IPK and PERK. In the context of lactation, however, transcriptional changes in the ER stress pathway at different stages of the lactation cycle are a normal aspect of the tissue's adaptation to the changing physiological state.
\end{abstract}

Key words: metabolism, dairy cow, transcriptomics

\footnotetext{
Received August 6, 2011.

Accepted November 16, 2011.

${ }^{1}$ Financial support provided by the Cooperative State Research, Education and Extension Service, US Department of Agriculture, under project No. ILLU-538-307.

${ }^{2}$ Corresponding author: jloor@illinois.edu
}

\section{Short Communication}

Recent studies of the bovine mammary gland have been aimed in particular at understanding gene networks involved in milk component biosynthesis and their interrelationships (Bionaz and Loor, 2008b; Harvatine et al., 2009; Bauman et al., 2011). Milk fat depression studies in laboratory animals (Bobrovnikova-Marjon et al., 2008) suggested a role of endoplasmic reticulum (ER) stress in the regulation of lipogenic pathways in mouse mammary epithelial cells (Invernizzi et al., 2010; Bauman et al., 2011). The ER can sense levels of particular intermediates or the redox state of thioldisulfide and pyridine nucleotide systems, which result from specific metabolic and nutritional conditions (Mandl et al., 2009), and activate signaling pathways or metabolic responses involving endocrine networks at the systemic level (Hotamisligil, 2010b) to restore homeostasis. In the cellular context, an impairment in protein folding leads to activation of the unfolded protein response (UPR), a signaling pathway operating between ER and nucleus aimed at increasing the clearance of misfolded proteins from the lumen of ER and reducing entry of new proteins (Rutkowski and Hegde, 2010). Maintenance of ER homeostasis, regulation of the UPR, or committing to apoptosis are strategies that cells can use to face different challenges that are often related to their nature (e.g., high secretory or metabolic capacity; Hotamisligil, 2010a).

In nonruminants, the ER transmembrane transcription factors activating transcription factor 6 (ATF6) and the RNA-dependent protein kinase (PKR)-like ER kinase (PERK) are the main players in the UPR signaling cascade (Brewer and Diehl, 2000). The PERK inhibits cellular proliferation pathways by blocking translation or indirectly inducing apoptosis by activating ATF4 (Harding et al., 1999). X-box binding protein 1 (XBP1) is another downstream component of a third inducer of the UPR pathway, ER to nucleus signaling 1 (IRE-1; Zeng et al., 2009). Upon nuclear translocation of ATF4 and ATF6, DNA damage-inducible transcript 3 protein (CCAAT/enhancer-binding protein homolo- 
gous protein, CHOP) is activated and leads to apoptosis.

Besides its primary role of maintaining cellular protein homeostasis, the UPR was recently shown to play a role in maintaining lipid homeostasis in mouse mammary epithelial cells through regulation of sterol regulatory element binding protein (SREBF) and its target proteins (Bobrovnikova-Marjon et al., 2008). The membrane-bound transcription factor peptidase, site 1 (MBTPS1) is another transcription factor in the ER stress pathway with a central role in regulation of lipid metabolism (e.g., activates SREBF). Watanabe et al. (2001) observed and proposed an important role for chaperone heat shock protein $90 \mathrm{kDa} \beta 1$, member 1 (HSP90B1/GRP94) in murine milk protein secretion. The above data in nonruminants suggest that the ER stress and UPR signaling components might be involved in the response to the pseudo metabolic stress state that accompanies the sudden and robust initiation and maintenance of synthesis and secretion of large amounts of milk protein and lipid during lactation (Ou et al., 2008). This idea is also supported by recent observations that UPR signaling directly targets genes involved in metabolic pathways both in physiological and non-physiological conditions (Hotamisligil, 2010b).

The hypothesis of the present study was that the mRNA expression of several components of the ER stress pathway changes from pregnancy through the different stages of lactation. Furthermore, such patterns of expression may be associated with the mRNA expression of metabolic genes and particularly those involved in milk fat synthesis regulation (Bionaz and Loor, 2008b). The experimental design has been thoroughly described elsewhere (Bionaz and Loor, 2007). In brief, percutaneous mammary biopsies were performed on $\mathrm{d}-15,1,15,60$, and 240 relative to parturition on 7 multiparous Holstein cows following protocols approved by the University of Illinois Institutional Animal Care and Use Committee. Mammary total RNA extraction and quantitative PCR were performed following procedures published previously (Bionaz and Loor, 2007). Ribosomal proteins S15 (RPS15) and S9 (RPS9) and ubiquitously expressed transcript isoform $2(U X T)$ were used as internal control genes for normalization (Bionaz and Loor, 2007). Messenger RNA expression of XBP1, ATF3, ATF 4, and ATF6, CHOP, MBTPS1, eukaryotic translation initiation factor $2 \alpha$ kinase 3 (Eif2ak3/ PERK), heat shock 70kDa protein 5 (HSPA5/GRPr8/ $B i P), G R P 94$ and DnaJ (Hsp 40) homolog, subfamily C, member 3 (DNAJC3/P58IPK) was measured. Primers were designed and evaluated (Bionaz and Loor, 2008a) and the sequences used ( $\mathrm{F}$, forward; $\mathrm{R}$, reverse) were XBP1, F: 5'-GAGAGCGAAGCCAATGTGGTA-3';
R 5'-ACTGTGAATTCAGGGTGATCTTTCT-3'; ATF3, F 5'-CCTGCAGAAAGAGTCGGAGAA-3'; R 5'-GCAGGTTGAGCATGTATATCAAATG-3'; ATF4, F 5'-AGCACTTCAAACATCATGGGTTCT-3'; R 5'-CATCCTCCTTGCTGTTGTCTGA-3'; ATF6, F 5'-AGCCCTGATGGTGCTAACTGA-3'; R 5'-TTCATGATTTAACCTGAGAGATTCTGTT-3'; CHOP, F 5'-GAACCTGAGGAGAGAGTGTTCCA-3'; R 5'-AGTGACTCAGCTGCCATCTCTGT-3'; $M B$ TPS1, F; 5'-CAACGGTTGTGGAATATGAGTACATT-3'; R 5'-TGTCGATTGCACTGCTCTTCA-3'; $P E R K, \mathrm{~F}$ 5'-ATATGAGCCCGGAACAGATTCAT-3'; R 5'-AGTGCCGAACGGGTATAGTAATTC-3'; BiP, F 5'-GCCCTGTCTTCCCAACATCA-3'; R 5'-CAGGTCCATGTTTAGCTCTTCAAA-3'; GRP94, F 5'-CTGATGAACGATATCAAACCCATATG-3'; R 5'-GTCATCGCTTTCCTTTGAAAATG-3'; P58IPK, F 5'-TGCAGTAGATGGTGACCCTGATA-3'; R 5'-GGAAGTGCTGCTTTTGATTTGC-3'. The underlined nucleotides represent exon-exon junctions. Median cycle threshold $(\mathrm{Ct})$ values ranged between 21.2 and 26.6 and median $\Delta \mathrm{Ct}$ between 0.06 and 5.55. Slopes ranged between -2.99 and -3.63 with coefficient of determination above 0.990. Analysis was performed only on data points with studentized residuals $<2.5$. Normalized gene expression data were transformed to $n$ fold change relative to $\mathrm{d}-15$ and then log-transformed (Bionaz and Loor, 2011).

The MIXED procedure of SAS (SAS Inst. Inc, Cary, $\mathrm{NC}$ ) with repeated measures was used to determine the overall effect of time on gene expression. The fixed effect considered in the model was time, and cow represented the random effect. Compound symmetry was used as covariate structure for repeated measures. Orthogonal polynomial contrasts were used to test for linear and quadratic effects over time. Quantitative PCR data are presented as $\log 2$ transformed relative to $-15 \mathrm{~d}$. Gene expression network and pathway analysis was performed using Ingenuity Pathway Analysis (Ingenuity Systems Inc., Mountain View, CA). Table 1 lists relative mRNA expression of all of the genes measured in this study. The expression of all genes except $X B P 1$, ATF6, MBTPS1, and GRP94 followed a significant ( $P$ $<0.07)$ quadratic response between $-15 \mathrm{~d}$ and established lactation (Table 1). Thus, the expression of affected genes resembled closely the curve of lactation in these same cows (Bionaz and Loor, 2007). Expression of $P 58 I P K$ during lactation reached a peak $(P=0.001)$ on $\mathrm{d} 60$ vs. $-15 \mathrm{~d}$ and slightly decreased $(P=0.006)$ by d 240;ATF3 (time $P=0.004)$ and $C H O P(P=0.06)$ shared a common expression pattern that peaked at $\mathrm{d}$ 15 , followed by a decrease through d 240. Expression of $\operatorname{PERK}(P=0.13)$ increased during lactation compared 
Table 1. Relative expression ${ }^{1}$ of genes (to $\mathrm{d}-15$ ) involved in the endoplasmic reticulum stress pathway during the lactation cycle

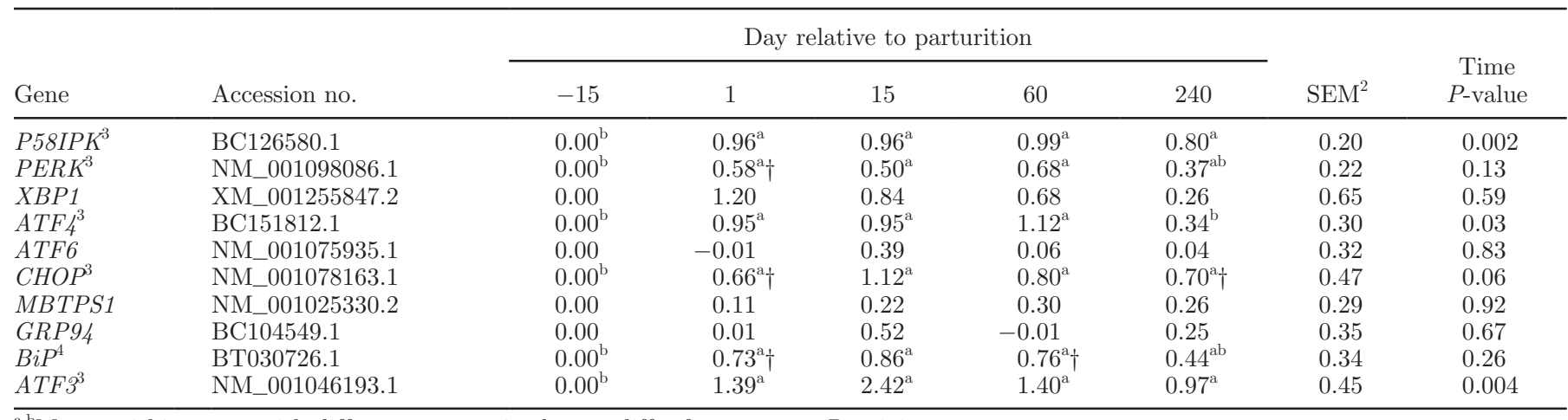

${ }_{\mathrm{a}, \mathrm{b}}$ Means within a row with different superscript letters differ from -15 at $P<0.05$.

${ }^{1}$ Fold of $d-15$ (log-transformed mRNA abundance).

${ }^{2}$ Highest value among time points.

${ }^{3}$ Significant $(P<0.07)$ quadratic response.

${ }^{4}$ Quadratic response $(P=0.12)$.

$\dagger$ Differs from $\mathrm{d}-15$ at $P<0.09$.

with $\mathrm{d}-15$ with peak values at $\mathrm{d} 60$. In a similar way, ATF 4 expression $(P=0.03)$ followed the same trend in the first $60 \mathrm{~d}$ postpartum (Table 1 ).

The role of ER stress signaling in inflammation and various metabolic processes (including disease states) in nonruminants is well known (Hotamisligil, 2010b). High rates of cellular biosynthetic activity are a characteristic of the bovine mammary gland during lactation, and especially during the 2 mo immediately after parturition (Bionaz and Loor, 2008b, 2011). Previous work in nonruminants has shown that lipogenesis in the mammary gland and liver is sensitive to ER stress, and adaptations in this pathway are closely regulated by PERK and XBP1 (Hotamisligil, 2010b); P58IPK inhibits PERK (Figure 1) and we found that its expression pattern was similar to that observed in murine mammary gland during pregnancy and lactation $[\mathrm{Ru}-$ dolph et al., 2007; i.e., lowest expression in the middle of pregnancy with a gradual increase as the animal approaches parturition, a peak (10-fold vs. pregnancy) in the first day of lactation, and a decrease thereafter (3.7-fold at d 9 vs. pregnancy)]. Milk production in mice peaks between 9 and $13 \mathrm{~d}$ of lactation (i.e., at midlactation; Rutkowski and Hegde, 2010), which suggests that in bovines, the expression of P58IPK was not strictly related to milk production.

The pattern of PERK expression with highest expression at 1 through $60 \mathrm{~d}$ postpartum was closely associated with the induction of lipogenic genes that we reported previously in these cows (Bionaz and Loor, 2008b). In mice, however, PERK transcription was greater before parturition and decreased slightly during the first part of lactation (Rudolph et al., 2007). The greater expression of ATF4 at d 60 (Figure 1), followed by a reduction in expression, did not correspond to its reported role in mice (Bagheri-Yarmand et al., 2003; i.e., it inhibits mammary epithelial cell proliferation and the lactogenic capacity of the tissue; Bagheri-Yarmand et al., 2003). The expression pattern of ATF 4 during the normal course of lactation in mice was characterized by a peak at $\mathrm{d} 1$, followed by a decrease at $\mathrm{d} 2$ and an additional increase by d 9 (Rudolph et al., 2007). The pattern of expression that we observed for ATF 4 could be related with the well-established progression in the rate of apoptosis relative to proliferation that characterizes the bovine mammary gland as lactation progresses (Capuco et al., 2001).

Hepatic lipogenesis in mice requires XBP1, which regulates expression of critical genes involved in fatty acid synthesis (Lee et al., 2008). Although not statistically significant due to the large degree of variation, expression of XBP1 peaked at d 1 and decreased gradually thereafter, which resembled closely the pattern of expression in murine mammary tissue [i.e., expression of $X B P 1$ increased approaching parturition and similarly peaked ( 2.5 fold) at d 1 of lactation compared with d 12 of gestation; Rudolph et al., 2007]. Although clear differences exist in the substrates used by the bovine and murine mammary gland for lipogenesis (e.g., acetate vs. glucose), at least for bovines, the pattern of $X B P 1$ expression we observed between $\mathrm{d}-15$ and 1 resembled that of SREBF1, PPARG, and several target genes related to milk fat synthesis that we reported previously in these same cows (Bionaz and Loor, 2008b). The observation that XBP1 expression decreased gradually after parturition could indicate that it may not be essential to sustain mammary gland lipogenesis; alternatively, it could mean that lipogenic gene expression is 


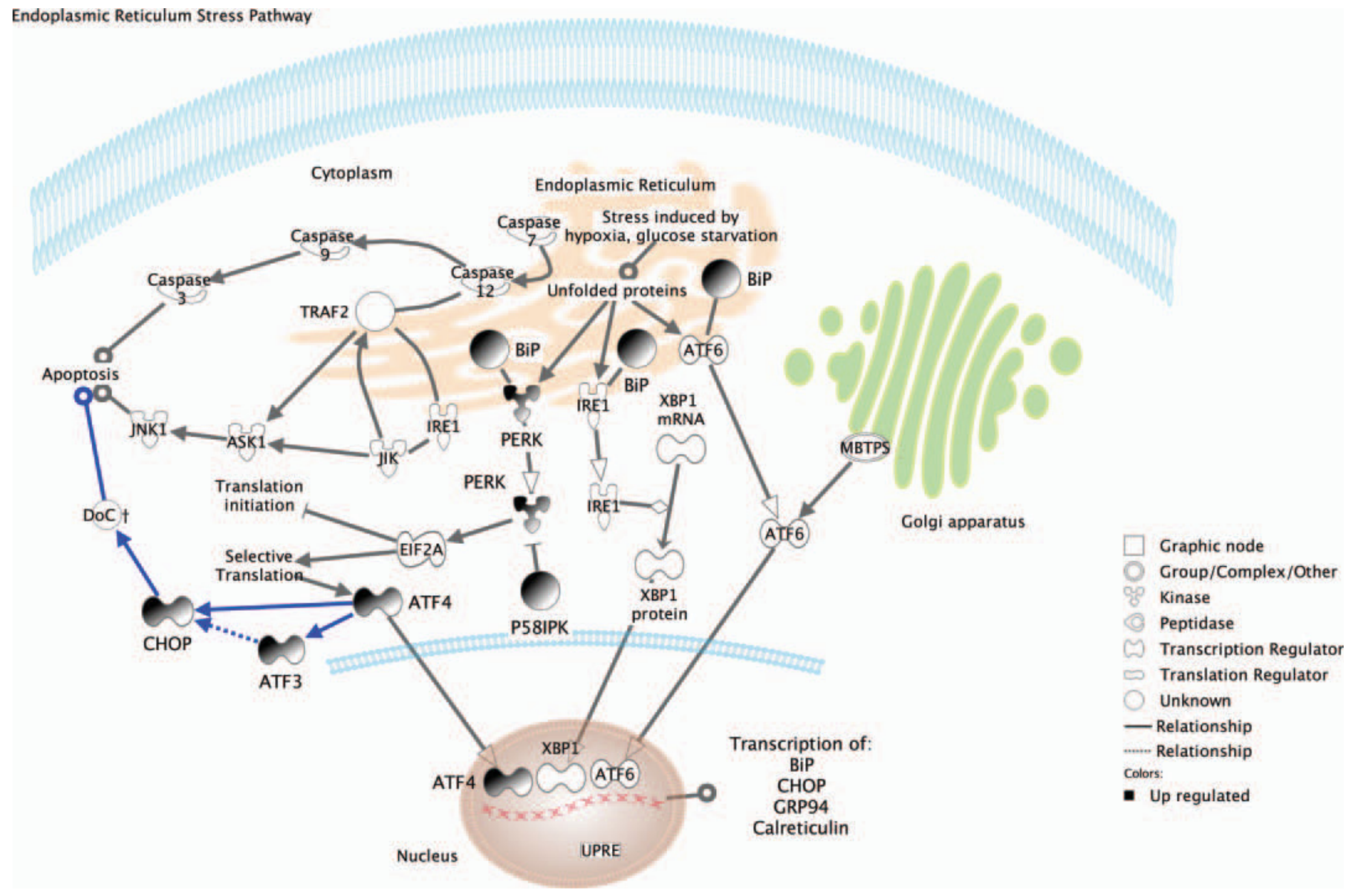

Figure 1. Mammary mRNA expression of components of the endoplasmic reticulum stress pathway at d 60 of lactation compared with $\mathrm{d}$ -15. Genes with darker background were upregulated. Pathway created with Ingenuity Pathway Analysis (Ingenuity Systems Inc., Mountain View, CA) using its knowledge base and the scientific literature. Color version available in the online PDF.

primarily under the control of other transcription regulators such as peroxisome proliferator-activated receptor $\gamma$ (PPAR $\gamma$; Bionaz and Loor, 2008b; Kadegowda et al., 2009). The study of Bionaz and Loor (2008b) showed that mammary tissue expression of PPAR $\gamma$ and several of its lipogenic target genes increased markedly at parturition and remained elevated during the course of lactation, which coincided with the curve of lactation. A functional link between PPAR $\gamma$ and mammary lipogenic gene expression was later shown in vitro with bovine mammary cells (Kadegowda et al., 2009).

Due to their involvement in apoptosis in nonruminant cells (Figure 1), downregulation (quadratic effect) of $\operatorname{BiP}(P=0.123), A T F 3(P=0.021)$, and $C H O P(P=$ $0.071)$ from 15 to $240 \mathrm{~d}$ (i.e., late lactation) was unexpected given that relative rates of apoptosis in the bovine mammary gland are greater as lactation progresses (Capuco et al., 2001). Rudolph et al. (2007) observed no changes in $C H O P$ mRNA expression during lactation in mice, whereas, ATF3 was greater during pregnancy and then decreased during the first $9 \mathrm{~d}$ of lactation. Expression of BiP peaked at parturition and decreased until reaching prepartal levels on d 9 (Rudolph et al., 2007). Although lipogenic gene expression in our cows by late lactation was lower than at early stages (Bionaz and Loor, 2008b), expression remained elevated relative to the nonlactating period (e.g., acetyl-CoA carboxylase was $\sim 3$-fold greater and stearoyl-CoA desaturase was $\sim 6$-fold greater at $240 \mathrm{~d}$ vs. $-15 \mathrm{~d}$ relative to parturition). Therefore, the gradual decrease in $C H O P$ expression we observed may represent a functional mechanism to control mRNA abundance of metabolic genes [e.g., mice lacking $C H O P$ and challenged with tunicamycin (i.e., an inducer of UPR) maintained normal rates of hepatic lipogenesis, leading to the notion that CHOP activity is partly responsible for reduced gene expression under severe ER stress (Hotamisligil, 2010b)].

Our results support previous findings in nonruminants (Bobrovnikova-Marjon et al., 2008; Lee et al., 2008; Hotamisligil, 2010b) showing that the ER stress- 
signaling pathway may be associated with mammary lipogenesis during lactation in dairy cows. For example, greater PERK expression at peak lactation (d 60) might be linked with milk fat synthesis, whereas greater expression of $C H O P$ in late lactation versus prepartum may be functionally related with the involution process after peak lactation. Regarding the overall process of milk synthesis, murine data (Rudolph et al., 2007) also provide some support for the idea that ER stress-related genes in bovine mammary tissue could be involved in milk protein synthesis because we observed previously in these same cows an upregulation of genes playing a central role in amino acid and glucose transport, insulin signaling, and also components of the mammalian target of rapamycin (mTOR) pathway in the first weeks postpartum (Bionaz and Loor, 2011). Whether the observed responses also occur in mammary tissue of beef cows, for example, remains to be established; however, it is likely that the genes we studied also would be upregulated during lactation (along with synthesis of fat and protein) but to a lesser degree and due, in part, to the lower milk production capacity (e.g., 11.2 vs. 8.6 $\mathrm{kg} / \mathrm{d}$ in late and early lactation; Radunz et al., 2010).

\section{ACKNOWLEDGMENTS}

We gratefully acknowledge the help from the staff of the University of Illinois Dairy Research and Teaching Unit for animal care.

\section{REFERENCES}

Bagheri-Yarmand, R., R. K. Vadlamudi, and R. Kumar. 2003. Activating transcription factor 4 overexpression inhibits proliferation and differentiation of mammary epithelium resulting in impaired lactation and accelerated involution. J. Biol. Chem. 278:17421-17429.

Bauman, D. E., K. J. Harvatine, and A. L. Lock. 2011. Nutrigenomics, rumen-derived bioactive fatty acids, and the regulation of milk fat synthesis. Annu. Rev. Nutr. 31:299-319.

Bionaz, M., and J. J. Loor. 2007. Identification of reference genes for quantitative real-time PCR in the bovine mammary gland during the lactation cycle. Physiol. Genomics 29:312-319.

Bionaz, M., and J. J. Loor. 2008a. ACSL1, AGPAT6, FABP3, LPIN1, and SLC27A6 are the most abundant isoforms in bovine mammary tissue and their expression is affected by stage of lactation. J. Nutr. 138:1019-1024.

Bionaz, M., and J. J. Loor. 2008b. Gene networks driving bovine milk fat synthesis during the lactation cycle. BMC Genomics 9:366.

Bionaz, M., and J. J. Loor. 2011. Gene networks driving bovine mammary protein synthesis during the lactation cycle. Bioinform. Biol. Insights 5:83-98.

Bobrovnikova-Marjon, E., G. Hatzivassiliou, C. Grigoriadou, M. Romero, D. R. Cavener, C. B. Thompson, and J. A. Diehl. 2008.
PERK-dependent regulation of lipogenesis during mouse mammary gland development and adipocyte differentiation. Proc. Natl. Acad. Sci. USA 105:16314-16319.

Brewer, J. W., and J. A. Diehl. 2000. PERK mediates cell-cycle exit during the mammalian unfolded protein response. Proc. Natl. Acad. Sci. USA 97:12625-12630.

Capuco, A. V., D. L. Wood, R. Baldwin, K. McLeod, and M. J. Paape. 2001. Mammary cell number, proliferation, and apoptosis during a bovine lactation: Relation to milk production and effect of bST. J. Dairy Sci. 84:2177-2187.

Harding, H. P., Y. Zhang, and D. Ron. 1999. Protein translation and folding are coupled by an endoplasmic-reticulum-resident kinase. Nature 397:271-274.

Harvatine, K. J., J. W. Perfield II, and D. E. Bauman. 2009. Expression of enzymes and key regulators of lipid synthesis is upregulated in adipose tissue during CLA-induced milk fat depression in dairy cows. J. Nutr. 139:849-854.

Hotamisligil, G. S. 2010a. Endoplasmic reticulum stress and atherosclerosis. Nat. Med. 16:396-399.

Hotamisligil, G. S. 2010b. Endoplasmic reticulum stress and the inflammatory basis of metabolic disease. Cell 140:900-917.

Invernizzi, G., B. J. Thering, M. A. McGuire, G. Savoini, and J. J. Loor. 2010. Sustained upregulation of stearoyl-CoA desaturase in bovine mammary tissue with contrasting changes in milk fat synthesis and lipogenic gene networks caused by lipid supplements. Funct. Integr. Genomics 10:561-575.

Kadegowda, A. K., M. Bionaz, L. S. Piperova, R. A. Erdman, and J. J. Loor. 2009. Peroxisome proliferator-activated receptor- $\gamma$ activation and long-chain fatty acids alter lipogenic gene networks in bovine mammary epithelial cells to various extents. J. Dairy Sci. 92:4276-4289.

Lee, A.-H., E. F. Scapa, D. E. Cohen, and L. H. Glimcher. 2008. Regulation of hepatic lipogenesis by the transcription factor XBP1. Science 320:1492-1496.

Mandl, J., T. Mészáros, G. Bánhegyi, L. Hunyady, and M. Csala. 2009. Endoplasmic reticulum: Nutrient sensor in physiology and pathology. Trends Endocrinol. Metab. 20:194-201.

Ou, L., Y. Wu, C. Ip, X. Meng, Y. C. Hsu, and M. M. Ip. 2008. Apoptosis induced by $t 10, c 12$-conjugated linoleic acid is mediated by an atypical endoplasmic reticulum stress response. J. Lipid Res. 49:985-994

Radunz, A. E., F. L. Fluharty, M. L. Day, H. N. Zerby, and S. C. Loerch. 2010. Prepartum dietary energy source fed to beef cows: I. Effects on pre- and postpartum cow performance. J. Anim. Sci. 88:2717-2728

Rudolph, M. C., J. L. McManaman, T. Phang, T. Russell, D. J. Kominsky, N. J. Serkova, T. Stein, S. M. Anderson, and M. C. Neville. 2007. Metabolic regulation in the lactating mammary gland: A lipid synthesizing machine. Physiol. Genomics 28:323-336.

Rutkowski, D. T., and R. S. Hegde. 2010. Regulation of basal cellular physiology by the homeostatic unfolded protein response. J. Cell Biol. 189:783-794.

Watanabe, A., I. Uchida, K. Nakata, Y. Fujimoto, and S. Oikawa. 2001. Molecular cloning of bovine (Bos taurus) cDNA encoding a 94-kDa glucose-regulated protein and developmental changes in its mRNA and protein content in the mammary gland. Comp. Biochem. Physiol. B Biochem. Mol. Biol. 130:547-557.

Zeng, L., A. Zampetaki, A. Margariti, A. E. Pepe, S. Alam, D. Martin, Q. Xiao, W. Wang, Z.-G. Jin, G. Cockerill, K. Mori, Y.-S. J. Li, Y. Hu, S. Chien, and Q. Xu. 2009. Sustained activation of XBP1 splicing leads to endothelial apoptosis and atherosclerosis development in response to disturbed flow. Proc. Natl. Acad. Sci. USA 106:8326-8331. 